\title{
Grand unification in the spectral Pati-Salam model
}

\author{
Ali H. Chamseddine, ${ }^{a, b}$ Alain Connes ${ }^{b, c, d}$ and Walter D. van Suijlekom ${ }^{e}$ \\ ${ }^{a}$ Physics Department, American University of Beirut, \\ Beirut, Lebanon \\ ${ }^{b}$ College de France, \\ 3 rue Ulm, F-75005 Paris, France \\ ${ }^{c}$ I.H.E.S., \\ F-91440 Bures-sur-Yvette, France \\ ${ }^{d}$ Department of Mathematics, The Ohio State University, \\ Columbus, OH 43210, U.S.A. \\ ${ }^{e}$ Institute for Mathematics, Astrophysics and Particle Physics, Radboud University Nijmegen, \\ Heyendaalseweg 135, 6525 AJ Nijmegen, The Netherlands \\ E-mail: chams@aub.edu.lb, alain@connes.org, waltervs@math.ru.nl
}

ABSTRACT: We analyze the running at one-loop of the gauge couplings in the spectral PatiSalam model that was derived in the framework of noncommutative geometry. There are a few different scenarios for the scalar particle content which are determined by the precise form of the Dirac operator for the finite noncommutative space. We consider these different scenarios and establish for all of them unification of the Pati-Salam gauge couplings. The boundary conditions are set by the usual RG flow for the Standard Model couplings at an intermediate mass scale at which the Pati-Salam symmetry is broken.

Keywords: Beyond Standard Model, Non-Commutative Geometry, Spontaneous Symmetry Breaking, Renormalization Group

ArXiv EPrint: 1507.08161 


\section{Contents}

1 Introduction 1

2 Spectral Pati-Salam and grand unification 3

2.1 Pati-Salam with composite Higgs fields 5

2.2 Pati-Salam with fundamental Higgs fields 8

2.3 Left-right symmetric Pati-Salam with fundamental Higgs fields 9

$\begin{array}{lll}3 & \text { Conclusions } & 10\end{array}$

\section{Introduction}

This paper builds on two recent discoveries in the noncommutative geometry approach to particle physics: we showed in [12] how to obtain inner fluctuations of the metric without having to assume the order one condition on the Dirac operator. Moreover the original argument by classification [6] of finite geometries $F$ that can provide the fine structure of Euclidean space-time as a product $M \times F$ (where $M$ is a usual 4-dimensional Riemannian space) has now been replaced by a much stronger uniqueness statement $[10,11]$. This new result shows that the algebra

$$
M_{2}(\mathbb{H}) \oplus M_{4}(\mathbb{C})
$$

where $\mathbb{H}$ are the quaternions, appears uniquely when writing the higher analogue of the Heisenberg commutation relations. This analogue is written in terms of the basic ingredients of noncommutative geometry where one takes a spectral point of view, encoding geometry in terms of operators on a Hilbert space $\mathcal{H}$. In this way, the inverse line element is an unbounded self-adjoint operator $D$. The operator $D$ is the tensor sum of the usual Dirac operator on $M$ and a 'finite Dirac operator' on $F$, which is simply a hermitian matrix $D_{F}$. The usual Dirac operator involves $\gamma$ matrices which allow one to combine the momenta into a single operator. The higher analogue of the Heisenberg relations puts the spatial variables on similar footing by combining them into a single operator $Y$ using another set of $\gamma$ matrices and it is in this process that the algebra (1.1) appears canonically and uniquely in dimension 4 . We refer to $[10,11]$ for a detailed account. What matters for the present paper is that the above process leads without arbitrariness to the Pati-Salam [22] gauge group $\mathrm{SU}(2)_{R} \times \mathrm{SU}(2)_{L} \times \mathrm{SU}(4)$, together with the corresponding gauge fields and a scalar sector, all derived as inner perturbations of $D$ [12]. Note that the scalar sector can not be chosen freely, in contrast to the early work on Pati-Salam unification $[3,13,15,16]$. In fact, there are only a few possibilities for the precise scalar content, depending on the assumptions made on the finite Dirac operator. 
From the spectral action principle, the dynamics and interactions are described by the spectral action $[4,5]$,

$$
\operatorname{tr}\left(f\left(D_{A} / \Lambda\right)\right)
$$

where $\Lambda$ is a cutoff scale and $f$ an even and positive function. In the present case, it can be expanded using heat kernel methods,

$$
\operatorname{tr}\left(f\left(D_{A} / \Lambda\right)\right) \sim F_{4} \Lambda^{4} a_{0}+F_{2} \Lambda^{2} a_{2}+F_{0} a_{4}+\cdots
$$

where $F_{4}, F_{2}, F_{0}$ are coefficients related to the function $f$ and $a_{k}$ are Seeley deWitt coefficients, expressed in terms of the curvature of $M$ and (derivatives of) the gauge and scalar fields. This action is interpreted as an effective field theory for energies lower than $\Lambda$.

One important feature of the spectral action is that it gives the usual Pati-Salam action with unification of the gauge couplings [12] (cf. eq. (3.1) below). This is very similar to the case of the spectral Standard Model [9] where there is unification of gauge couplings. Since it is well known that the SM gauge couplings do not meet exactly, it is crucial to investigate the running of the Pati-Salam gauge couplings beyond the Standard Model and to find a scale $\Lambda$ where there is grand unification:

$$
g_{R}(\Lambda)=g_{L}(\Lambda)=g(\Lambda)
$$

This would then be the scale at which the spectral action (1.3) is valid as an effective theory. There is a hierarchy of three energy scales: SM, an intermediate mass scale $m_{R}$ where symmetry breaking occurs and which is related to the neutrino Majorana masses $\left(10^{11}-10^{13} \mathrm{GeV}\right)$, and the GUT scale $\Lambda$.

For simplicity, we restrict our analysis to the running of the gauge couplings at one loop. Indeed, at two loops the gauge and scalar couplings are mixed and influence each other. Hence at the two loop level the running of the gauge couplings requires a complete understanding of the scalar sector and the scalar potential, which in turn requires a suitable method for dealing with quadratic divergences that appear from the mass terms. This deserves a careful study and goes beyond the aims of the present paper.

Thus, we analyze the running of the gauge couplings according to the usual (one-loop) $\mathrm{RG}$ equation where each takes the form

$$
16 \pi^{2} \frac{d g}{d t}=-b g^{3}
$$

The coefficient $b$ is determined by the particle content and their representation theory [14, 17-19] for which we use [20] as well as the program PyR@TE. As mentioned before, depending on the assumptions on $D_{F}$, one may vary to a limited extent the scalar particle content, consisting of either composite or fundamental scalar fields. We will not limit ourselves to a specific model but consider all cases separately. In fact, we establish grand unification for all of them, thus confirming validity of the spectral action at the corresponding scale, independent of the specific form of $D_{F}$.

Shortly after the submission of the present paper, the preprint [2] appeared in which our results are confirmed and confronted with recent data from LHC. 


\section{Spectral Pati-Salam and grand unification}

One of the pressing questions at present is whether there is new physics beyond the Standard Model. The success of the spectral construction of the Standard Model, predicting its particle content, including gauge fields, Higgs fields as well as a singlet whose vev gives Majorana mass to the right handed neutrino, is a strong signal that we are on the right track. The route that led to this conclusion starts with classifying the algebras of the finite space. The results show that the only algebras which solve the fermion doubling problem are of the form $M_{2 a}(\mathbb{C}) \oplus M_{2 a}(\mathbb{C})$ where $a$ is an even integer. An arbitrary symplectic constraint is imposed on the first algebra restricting it from $M_{2 a}(\mathbb{C})$ to $M_{a}(\mathbb{H})$. The first non-trivial algebra one can consider is for $a=2$ with the algebra [6]

$$
M_{2}(\mathbb{H}) \oplus M_{4}(\mathbb{C}) .
$$

Coincidentally, and as explained in the introduction, the above algebra comes out as a solution of the two-sided Heisenberg quantization relation between the Dirac operator $D$ and the two maps from the four spin-manifold and the two four spheres $S^{4} \times S^{4}[10,11]$. This removes the arbitrary symplectic constraint and replaces it with a relation that quantizes the four-volume in terms of two quanta of geometry and have far reaching consequences on the structure of space-time.

The existence of the chirality operator $\gamma$ that commutes with the algebra breaks the quaternionic matrices $M_{2}(\mathbb{H})$ to the diagonal subalgebra and leads us to consider the finite algebra

$$
\mathcal{A}_{F}=\mathbb{H}_{R} \oplus \mathbb{H}_{L} \oplus M_{4}(\mathbb{C}) .
$$

The Pati-Salam gauge group $\mathrm{SU}(2)_{R} \times \mathrm{SU}(2)_{L} \times \mathrm{SU}(4)$ is obtained as the inner automorphism group of $\mathcal{A}=\mathcal{C}^{\infty}(M) \otimes \mathcal{A}_{F}$, and the corresponding gauge bosons appear as inner perturbations of the (spacetime) Dirac operator [12].

Next, an element of the Hilbert space $\Psi \in \mathcal{H}$ is represented by

$$
\Psi_{M}=\left(\begin{array}{c}
\psi_{A} \\
\psi_{A^{\prime}}
\end{array}\right), \quad \psi_{A^{\prime}}=\psi_{A}^{c}
$$

where $\psi_{A}^{c}$ is the conjugate spinor to $\psi_{A}$. Thus all primed indices $A^{\prime}$ correspond to the Hilbert space of conjugate spinors. It is acted on by both the left algebra $M_{2}(\mathbb{H})$ and the right algebra $M_{4}(\mathbb{C})$. Therefore the index $A$ can take 16 values and is represented by

$$
A=\alpha I
$$

where the index $\alpha$ is acted on by quaternionic matrices and the index $I$ by $M_{4}(\mathbb{C})$ matrices. Moreover, when the grading breaks $M_{2}(\mathbb{H})$ into $\mathbb{H}_{R} \oplus \mathbb{H}_{L}$ the index $\alpha$ is decomposed to $\alpha=\dot{a}, a$ where $\dot{a}=\dot{1}, \dot{2}$ (dotted index) is acted on by the first quaternionic algebra $\mathbb{H}_{R}$ and $a=1,2$ is acted on by the second quaternionic algebra $\mathbb{H}_{L}$. When $M_{4}(\mathbb{C})$ breaks into $\mathbb{C} \oplus M_{3}(\mathbb{C}$ ) (due to symmetry breaking or through the use of the order one condition as in [6]) the index $I$ is decomposed into $I=1, i$ and thus distinguishing leptons and quarks, 
where the 1 is acted on by the $\mathbb{C}$ and the $i$ by $M_{3}(\mathbb{C})$. Therefore the various components of the spinor $\psi_{A}$ are

$$
\begin{array}{rlrl}
\psi_{\alpha I} & =\left(\begin{array}{cccc}
\nu_{R} & u_{i R} & \nu_{L} & u_{i L} \\
e_{R} & d_{i R} & e_{L} & d_{i L}
\end{array}\right), & i=1,2,3 & \\
& =\left(\psi_{\dot{a} 1}, \psi_{a i}, \psi_{a 1}, \psi_{a i}\right), & a=1,2, \quad \dot{a}=\dot{1}, \dot{2}
\end{array}
$$

This is a general prediction of the spectral construction that there is 16 fundamental Weyl fermions per family, 4 leptons and 12 quarks.

The (finite) Dirac operator can be written in matrix form

$$
D_{F}=\left(\begin{array}{cc}
D_{A}^{B} & D_{A}^{B^{\prime}} \\
D_{A^{\prime}}^{B} & D_{A^{\prime}}^{B^{\prime}}
\end{array}\right)
$$

and must satisfy the properties

$$
\gamma_{F} D_{F}=-D_{F} \gamma_{F} \quad J_{F} D_{F}=D_{F} J_{F}
$$

where $J_{F}^{2}=1$. Matrix realizations of $\gamma_{F}$ and $J_{F}$ are given by

$$
\gamma_{F}=\left(\begin{array}{cc}
G_{F} & 0 \\
0 & -\bar{G}_{F}
\end{array}\right), \quad G_{F}=\left(\begin{array}{cc}
1_{2} & 0 \\
0 & -1_{2}
\end{array}\right), \quad J_{F}=\left(\begin{array}{cc}
0_{4} & 1_{4} \\
1_{4} & 0_{4}
\end{array}\right) \circ \mathrm{cc}
$$

where cc stands for complex conjugation. These relations, together with the hermiticity of $D$ imply the relations

$$
\left(D_{F}\right)_{A^{\prime}}^{B^{\prime}}=\left(\bar{D}_{F}\right)_{A}^{B} \quad\left(D_{F}\right)_{A^{\prime}}^{B}=\left(\bar{D}_{F}\right)_{B}^{A^{\prime}}
$$

and have the following zero components [7]

$$
\begin{gathered}
\left(D_{F}\right)_{a I}^{b J}=0=\left(D_{F}\right)_{\dot{a} I}^{\dot{b} J} \\
\left(D_{F}\right)_{a I}^{\dot{b}^{\prime} J^{\prime}}=0=\left(D_{F}\right)_{\dot{a} I}^{b^{\prime} J \prime}
\end{gathered}
$$

leaving the components $\left(D_{F}\right)_{a I}^{\dot{b} J},\left(D_{F}\right)_{a I}^{b^{\prime} J^{\prime}}$ and $\left(D_{F}\right)_{\dot{a} I}^{\dot{b}^{\prime} J^{\prime}}$ arbitrary. These restrictions lead to important constraints on the structure of the connection that appears in the inner fluctuations of the Dirac operator. In particular the operator $D$ of the full noncommutative space given by

$$
D=D_{M} \otimes 1+\gamma_{5} \otimes D_{F}
$$

gets modified to

$$
D_{A}=D+A_{(1)}+J A_{(1)} J^{-1}+A_{(2)}
$$

where

$$
A_{(1)}=\sum a[D, b], \quad A_{2}=\sum \widehat{a}\left[A_{(1)}, \widehat{b}\right], \quad \widehat{a}=J a J^{-1}
$$


We have shown in [12] that components of the connection $A$ which are tensored with the Clifford gamma matrices $\gamma^{\mu}$ are the gauge fields of the Pati-Salam model with the symmetry of SU $(2)_{R} \times \mathrm{SU}(2)_{L} \times \mathrm{SU}(4)$. On the other hand, the non-vanishing components of the connection which are tensored with the gamma matrix $\gamma_{5}$ are given by

$$
(A)_{a I}^{\dot{b} J} \equiv \gamma_{5} \Sigma_{a I}^{\dot{b} J}, \quad(A)_{a I}^{b^{\prime} J^{\prime}}=\gamma_{5} H_{a I b J}, \quad(A)_{\dot{a} I}^{\dot{b}^{\prime} J^{\prime}} \equiv \gamma_{5} H_{\dot{a} I \dot{b} J}
$$

where $H_{a I b J}=H_{b J a I}$ and $H_{\dot{a} I \dot{b} J}=H_{\dot{b} J a I}$, which is the most general Higgs structure possible. These correspond to the representations with respect to $\mathrm{SU}(2)_{R} \times \mathrm{SU}(2)_{L} \times \mathrm{SU}(4)$ :

$$
\begin{aligned}
\sum_{a I}^{\dot{b} J} & =\left(2_{R}, 2_{L}, 1\right)+\left(2_{R}, 2_{L}, 15\right) \\
H_{a I b J} & =\left(1_{R}, 1_{L}, 6\right)+\left(1_{R}, 3_{L}, 10\right) \\
H_{\dot{a} I \dot{I} J} & =\left(1_{R}, 1_{L}, 6\right)+\left(3_{R}, 1_{L}, 10\right)
\end{aligned}
$$

We note, however, that the inner fluctuations form a semi-group and if a component $\left(D_{F}\right)_{a I}^{\dot{b} J}$ or $\left(D_{F}\right)_{a I}^{b^{\prime} J^{\prime}}$ or $\left(D_{F}\right)_{\dot{a} I}^{\dot{b}^{\prime} J^{\prime}}$ vanish, then the corresponding $A$ field will also vanish. We distinguish three cases: 1) Left-right symmetric Pati-Salam model with fundamental Higgs fields $\Sigma_{a I}^{\dot{b} J}, H_{a I b J}$ and $H_{\dot{a} I \dot{b} J}$. In this model the field $H_{a I b J}$ should have a zero vev. 2) A Pati-Salam model where the Higgs field $H_{a I b J}$ that couples to the left sector is set to zero which is desirable because there is no symmetry between the left and right sectors at low energies. 3) If one starts with $\left(D_{F}\right)_{a I}^{\dot{b} J}$ or $\left(D_{F}\right)_{a I}^{b^{\prime} J^{\prime}}$ or $\left(D_{F}\right)_{\dot{a} I}^{\dot{b}^{\prime} J^{\prime}}$ whose values are given by those that were derived for the Standard Model, then the Higgs fields $\Sigma_{a I}^{\dot{b} J}, H_{a I b J}$ and $H_{\dot{a} I \dot{b} J}$ will become composite and expressible in terms of more fundamental fields $\Sigma_{I}^{J}, \Delta_{\dot{a} J}$ and $\phi_{\dot{a}}^{b}$. We refer to this as the composite model.

Depending on the precise particle content we determine the coefficients $b_{R}, b_{L}, b$ in (1.5) that control the RG flow of the Pati-Salam gauge couplings $g_{R}, g_{L}, g$. We run them to look for unification of the coupling $g_{R}=g_{L}=g$. The boundary conditions are taken at the intermediate mass scale $\mu=m_{R}$ to be the usual (e.g. [21, eq. (5.8.3)])

$$
\frac{1}{g_{1}^{2}}=\frac{2}{3} \frac{1}{g^{2}}+\frac{1}{g_{R}^{2}}, \quad \frac{1}{g_{2}^{2}}=\frac{1}{g_{L}^{2}}, \quad \frac{1}{g_{3}^{2}}=\frac{1}{g^{2}},
$$

in terms of the Standard Model gauge couplings $g_{1}, g_{2}, g_{3}$. At the mass scale $m_{R}$ the Pati-Salam symmetry is broken to that of the Standard Model, and we take it to be the same scale that is present in the see-saw mechanism. It should thus be of the order $10^{11}-10^{13} \mathrm{GeV}$. We now discuss the three models, in order of complexity.

\subsection{Pati-Salam with composite Higgs fields}

We first consider the case of a finite Dirac operator for which the Standard Model subalgebra $\mathbb{C} \oplus \mathbb{H}_{L} \oplus M_{3}(\mathbb{C}) \subset \mathcal{A}_{F}$ satisfies the first-order condition [6]. This condition is extremely constraining and forces the couplings of the right-handed neutrino to be with a singlet. In this case, the off-diagonal term in (2.6) becomes

$$
D_{\alpha I}^{\beta^{\prime} K^{\prime}}=\delta_{\alpha}^{i} \delta_{1^{\prime}}^{\beta^{\prime}} \delta_{I}^{1} \delta_{1^{\prime}}^{K^{\prime}} k^{* \nu_{R}},
$$




\begin{tabular}{|c|c|c|c|}
\hline particle & \multicolumn{3}{|c|}{$\mathrm{SU}(2)_{R} \mathrm{SU}(2)_{L} \mathrm{SU}(4)$} \\
\hline$\phi_{\dot{a}}^{b}$ & 2 & 2 & 1 \\
\hline$\Delta_{\dot{a} I}$ & 2 & 1 & 4 \\
\hline$\Sigma_{J}^{I}$ & 1 & 1 & 15 \\
\hline
\end{tabular}

Table 1. Pati-Salam scalar particle content and their representations for a first-order Dirac operator. The field $\Sigma_{J}^{I}$ in the last row is decoupled if there is quark-lepton coupling unification.

and the diagonal structure of $D_{F}$ is determined by the following sub-matrices [7]

$$
\begin{aligned}
D_{\alpha 1}^{\beta 1} & =\left(\begin{array}{cc}
0 & D_{a 1}^{\dot{b} 1} \\
D_{\dot{a} 1}^{b 1} & 0
\end{array}\right), & D_{a 1}^{\dot{b} 1}=\left(D_{\dot{a} 1}^{b 1}\right)^{*} \equiv D_{a(l)}^{\dot{b}} \\
D_{\alpha i}{ }^{\beta j} & =\left(\begin{array}{cc}
0 & D_{a(q)}^{\dot{b}} \delta_{i}^{j} \\
D_{\dot{a}(q)}^{b} \delta_{i}^{j} & 0
\end{array}\right), & D_{\dot{a}(q)}^{b}=\left(D_{a(q)}^{\dot{b}}\right)^{*}
\end{aligned}
$$

where

$$
D_{a(q)}^{\dot{b}}=\left(\begin{array}{cc}
k^{* u} & 0 \\
0 & k^{* d}
\end{array}\right) .
$$

The Yukawa couplings $k^{\nu}, k^{e}, k^{u}, k^{d}$ are $3 \times 3$ matrices in generation space. Notice that this structure gives Dirac masses to all the fermions, but Majorana masses only for the righthanded neutrinos. One can also consider the special case of lepton and quark unification by equating $k^{\nu}=k^{u}, k^{e}=k^{d}$ which imply some simplifications.

The inner perturbations of the finite Dirac operator of the above type were determined in [12] to be composite fields $\Sigma_{\dot{a} I}^{b J}$ and $H_{\dot{a} I \dot{b} J}$, depending on fundamental Higgs fields $\phi_{\dot{a}}^{b}$, $\Sigma_{I}^{J}$ and $\Delta_{\dot{a} J}$ in the following way:

$$
\begin{aligned}
\Sigma_{\dot{a} I}^{b J} & =\left(k^{\nu} \phi_{\dot{a}}^{b}+k^{e} \widetilde{\phi}_{\dot{a}}^{b}\right) \Sigma_{I}^{J}+\left(k^{u} \phi_{\dot{a}}^{b}+k^{d} \widetilde{\phi}_{\dot{a}}^{b}\right)\left(\delta_{I}^{J}-\Sigma_{I}^{J}\right), \\
H_{\dot{a} I \dot{b} J} & =k^{* \nu_{R}} \Delta_{\dot{a} J} \Delta_{\dot{b} I} \cdot
\end{aligned}
$$

The field $\widetilde{\phi}_{\dot{a}}^{b}$ is not an independent field and is given by

$$
\widetilde{\phi}_{\dot{a}}^{b}=\sigma_{2} \bar{\phi}_{\dot{a}}^{b} \sigma_{2}
$$

We have listed the fundamental Higgs fields and their representations in table 1. We first assume that there is lepton quark unification, so that the $\Sigma_{I}^{J}$ is decoupled.

Before turning to the computation of the $\beta$-functions of the Pati-Salam gauge couplings for the composite model, let us discuss the scalar sector that remains after spontaneous symmetry breaking to the Standard Model gauge group. A quick analysis leads to the scalar fields listed in table 2. Note that this includes the SM Higgs and a real scalar singlet. The latter played a key role in [7] in lowering the Higgs mass prediction to a realistic value [8]. 


\begin{tabular}{|c|ccc|}
\hline & $\mathrm{U}(1)_{Y}$ & $\mathrm{SU}(2)_{L} \mathrm{SU}(3)$ \\
\hline$\left(\begin{array}{c}\phi_{1}^{0} \\
\phi_{1}^{+}\end{array}\right)=\left(\begin{array}{c}\phi_{\dot{1}}^{1} \\
\phi_{\dot{1}}^{2}\end{array}\right)$ & 1 & 2 & 1 \\
$\left(\begin{array}{c}\phi_{2}^{-} \\
\phi_{2}^{0}\end{array}\right)=\left(\begin{array}{c}\phi_{\dot{2}}^{1} \\
\phi_{\dot{2}}^{2}\end{array}\right)$ & -1 & 2 & 1 \\
$\sigma$ & 0 & 1 & 1 \\
$\eta$ & $-\frac{2}{3}$ & 1 & 3 \\
\hline
\end{tabular}

Table 2. Scalar particle content induced by the composite model with SM-representations.

A qualitative study of the form of the scalar potential that we have done for the present Pati-Salam composite model indicates that this result continues to hold here. However, being interested mainly in the running of the gauge couplings, we leave a full study of the potential and its physical implications for future work.

The presence of the above scalar fields of course also have an influence on the running of the Standard Model gauge couplings (at one loop). We compute that instead of the usual $\beta$-functions $\left(b_{1}, b_{2}, b_{3}\right)=\left(-\frac{41}{6}, \frac{19}{6}, 7\right)$ we have

$$
\left(b_{1}, b_{2}, b_{3}\right)=\left(-\frac{64}{9}, 3, \frac{41}{6}\right) .
$$

One observes that this difference is relatively small (less than 5\%). In fact, the scalar fields that appear in addition to the SM Higgs have a negligible effect in our study of the running of the gauge couplings below. Moreover, if some of the scalar fields have a mass of order $m_{R}$ or higher, they are decoupled [1]. Note that this is in contrast to the effect on the running of the scalar couplings where, as already mentioned, the additional fields significantly change the physics of the Higgs sector. As said, the full analysis lies beyond the scope of this paper, but we refer to [8] for the relevant example.

Next, we compute the $\beta$-functions for the Pati-Salam couplings $g_{R}, g_{L}, g$ in the presence of the above composite particle content (cf. table 1 ):

$$
\left(b_{R}, b_{L}, b\right)=\left(\frac{7}{3}, 3, \frac{31}{3}\right) .
$$

The solutions of the RG-equations are easily found to be

$$
\begin{aligned}
g_{R}(\mu)^{-2} & =g_{R}\left(m_{R}\right)^{-2}+\frac{1}{8 \pi^{2}} \frac{7}{3} \log \frac{\mu}{m_{R}}, \\
g_{L}(\mu)^{-2} & =g_{L}\left(m_{R}\right)^{-2}+\frac{1}{8 \pi^{2}} 3 \log \frac{\mu}{m_{R}}, \\
g(\mu)^{-2} & =g\left(m_{R}\right)^{-2}+\frac{1}{8 \pi^{2}} \frac{31}{3} \log \frac{\mu}{m_{R}},
\end{aligned}
$$

We impose the boundary conditions (2.19) at the mass scale $\mu=m_{R}$. 


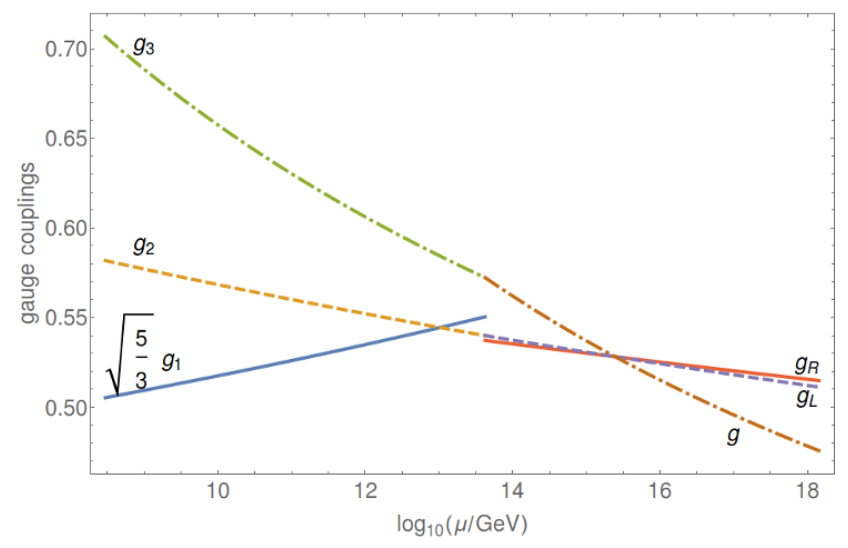

Figure 1. Running of coupling constants for the spectral Pati-Salam model with composite Higgs fields: $g_{1}, g_{2}, g_{3}$ for $\mu<m_{R}$ and $g_{R}, g_{L}, g$ for $\mu>m_{R}$ with unification scale $\Lambda \approx 2.5 \times 10^{15} \mathrm{GeV}$ for $m_{R}=4.25 \times 10^{13} \mathrm{GeV}$.

Note that in our analysis we have disregarded the non-renormalizable, order eight terms that appear in the expansion of the spectral action for the composite model [12, section 8], so let us argue why they can be ignored. In fact, since we consider only the running of the gauge couplings at the one loop level, we can safely ignore these non-renormalizable terms. Moreover, their contribution to the running of other (scalar) couplings will be suppressed by negative powers of $m_{R}$, at least at the one loop level.

Our approach for finding a unification scale is as follows. We search for an energy scale where the couplings $g_{R}, g_{L}$ and $g$ are equal by varying the scale $m_{R}$ at which the boundary conditions (2.19) are imposed. With the running of the Pati-Salam couplings governed by the coefficients (2.24) there is a unique value of $m_{R}$ for which the three lines meet. The unification scale is $\Lambda \approx 2.5 \times 10^{15} \mathrm{GeV}$ and the value found for the intermediate scale is $m_{R}=4.25 \times 10^{13} \mathrm{GeV}$ (figure 1 ).

If the scalar field $\Sigma_{I}^{J}$ is not decoupled - in other words, if there is no lepton-quark coupling unification - then there is an additional scalar $\left(1_{R}, 1_{L}, 15\right)$ irreducible representation contributing to the $\beta$-function, giving a slightly different $\left(b_{R}, b_{L}, b\right)=\left(\frac{7}{3}, 3,9\right)$. This in turn gives a unification scale $\Lambda \approx 6.3 \times 10^{15} \mathrm{GeV}$ for $m_{R}=4.1 \times 10^{13} \mathrm{GeV}$.

Let us conclude this subsection by mentioning that because of the assumptions made in our analysis, we trust these values only as indicative of the corresponding orders of magnitudes.

\subsection{Pati-Salam with fundamental Higgs fields}

Next, we consider the case of a more general finite Dirac operator, not satisfying the firstorder condition with respect to the Standard Model subalgebra. We begin with the special case where

$$
\left(D_{F}\right)_{a I}^{b^{\prime} J^{\prime}}=0
$$

which implies that the Higgs field $H_{a I b J}=0$. The inner perturbations $\Sigma_{\dot{a} I}^{b J}$ and $H_{\dot{a} I \dot{I} J}$ are now themselves fundamental Higgs fields [12, section 5] and their representations are listed in table 3 . 


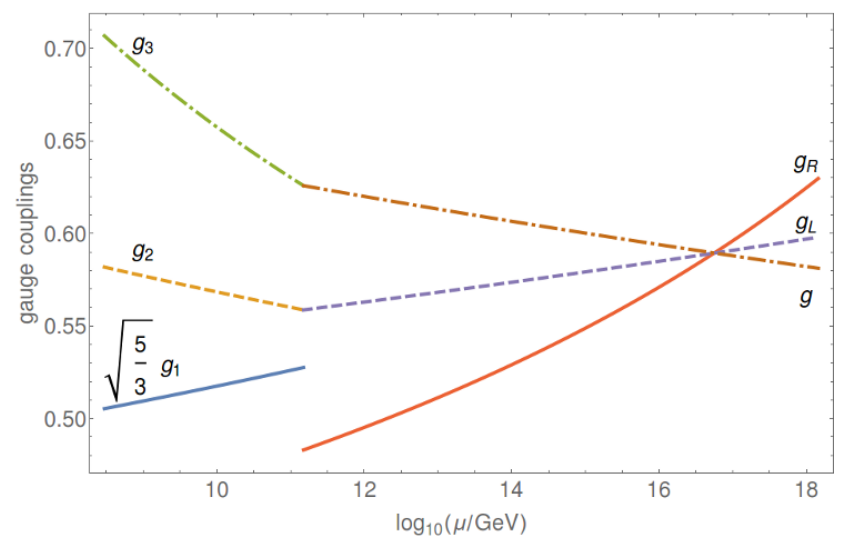

Figure 2. Running of coupling constants for the spectral Pati-Salam model with fundamental Higgs fields: $g_{1}, g_{2}, g_{3}$ for $\mu<m_{R}$ and $g_{R}, g_{L}, g$ for $\mu>m_{R}$ with unification scale $\Lambda \approx 6.3 \times 10^{16} \mathrm{GeV}$ for $m_{R}=1.5 \times 10^{11} \mathrm{GeV}$.

\begin{tabular}{|c|ccc|}
\hline particle & $\mathrm{SU}(2)_{R}$ & $\mathrm{SU}(2)_{L}$ & $\mathrm{SU}(4)$ \\
\hline$\sum_{\dot{a} J}^{b J}$ & 2 & 2 & $1+15$ \\
$H_{\dot{a} I \dot{b} J}\{$ & 3 & 1 & 10 \\
& 1 & 1 & 6 \\
\hline
\end{tabular}

Table 3. Pati-Salam scalar particle content and their representations for a general finite Dirac operator.

The contribution of the enlarged scalar sector on the running of the SM gauge couplings can be ignored if we assume that most of them are heavy (mass of the order $m_{R}$ ) so that we can apply the decoupling theorem of [1]. The remaining low-dimensional representations that describe the 'light' scalars have a small contribution to the running of the SM gauge couplings and can be safely ignored, also since we are mainly interested in the order of magnitudes for the intermediate scale and the unification scale.

With the scalar content of table 3 we determine the Pati-Salam $\beta$-functions to be

$$
\left(b_{R}, b_{L}, b\right)=\left(-\frac{26}{3},-2,2\right)
$$

Note that the $\mathrm{SU}(2)_{R}$ and $\mathrm{SU}(2)_{L}$-sectors are not asymptotically free, due to the large scalar sector. Nevertheless, we can still run the gauge couplings with the boundary values set by $(2.19)$.

Adopting the same approach as in the previous section for finding a unification scale, we arrive at figure 2 . The unification scale is $\Lambda \approx 6.3 \times 10^{16} \mathrm{GeV}$ if we set $m_{R}=1.5 \times 10^{11} \mathrm{GeV}$.

\subsection{Left-right symmetric Pati-Salam with fundamental Higgs fields}

As a final possibility we consider the most general case for $D_{F}$ which gives in addition to the fundamental Higgs fields in table 3 the field $H_{\text {aIbJ }}$ in the $\left(1_{R}, 3_{L}, 10\right)+\left(1_{R}, 1_{L}, 6\right)$ 


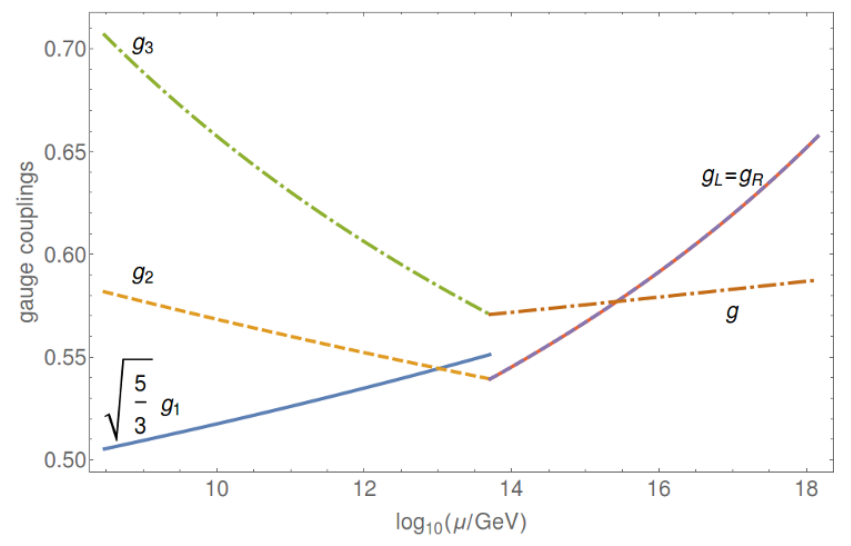

Figure 3. Running of coupling constants for the left-right symmetric spectral Pati-Salam model: $g_{1}, g_{2}, g_{3}$ for $\mu<m_{R}$ and $g_{R}, g_{L}, g$ for $\mu>m_{R}$ with unification scale $\Lambda \approx 2.7 \times 10^{15} \mathrm{GeV}$ for $m_{R}=5.1 \times 10^{13} \mathrm{GeV}$.

representation. The $\beta$-functions become

$$
\left(b_{R}, b_{L}, b\right)=\left(-\frac{26}{3},-\frac{26}{3},-\frac{4}{3}\right)
$$

Since we are again only interested in the order of magnitude for $m_{R}$ and $\Lambda$, we adopt the same approximations on the structure of the scalar sector as in the previous section. The scale $m_{R}$ is determined so as to have left-right symmetry $g_{R}\left(m_{R}\right)=g_{L}\left(m_{R}\right)$. This happens for $m_{R}=5.1 \times 10^{13} \mathrm{GeV}$, resulting in figure 3 . We find the unification scale to be $\Lambda \approx 2.7 \times 10^{15} \mathrm{GeV}$.

\section{Conclusions}

We have analyzed the running of the Pati-Salam gauge couplings for the spectral model, considering different scalar field contents corresponding to the assumptions made on the finite Dirac operator. We stress that the number of possible models is quite restrictive and that one can not freely choose the particle content. We have identified the three main models, although there exists small variations on them. The different possibilities correspond to restrictions on the geometry of the finite space $F$. In all the models considered here, we establish unification of the gauge couplings, with boundary conditions set by the usual Standard Model gauge couplings at an intermediate mass scale.

Besides the direct physical interest of such grand unification, it also determines the scale at which the asymptotic expansion of Equation (1.3) is actually valid as an effective theory. In order to see this, note that the scale-invariant term $F_{0} a_{4}$ in (1.3) for the spectral Pati-Salam model contains the terms [12]:

$$
\frac{F_{0}}{2 \pi^{2}} \int\left(g_{L}^{2}\left(W_{\mu \nu L}^{\alpha}\right)^{2}+g_{R}^{2}\left(W_{\mu \nu R}^{\alpha}\right)^{2}+g^{2}\left(V_{\mu \nu}^{m}\right)^{2}\right) .
$$

Normalizing this to give the Yang-Mills Lagrangian demands

$$
\frac{F_{0}}{2 \pi^{2}} g_{L}^{2}=\frac{F_{0}}{2 \pi^{2}} g_{R}^{2}=\frac{F_{0}}{2 \pi^{2}} g^{2}=\frac{1}{4},
$$


which requires gauge coupling unification, $g_{R}=g_{L}=g$. Note that the similar result for the Standard Model gauge couplings does not hold (at least at the one-loop level) because the three couplings actually do not meet, even though they are required to be unified in the spectral action [4]. We consider this to be strong evidence for the spectral Pati-Salam model as a realistic possibility to go beyond the Standard Model.

To summarize, the spectral construction of particle physics models based on a spectral triple with a noncommutative space with metric dimension four and whose finite part has KO dimension 6 leads directly to a family of Pati-Salam models with gauge symmetry $\mathrm{SU}(2)_{R} \times \mathrm{SU}(2)_{L} \times \mathrm{SU}(4)$ and well-defined Higgs structure. Breaking of SU $(2)_{R} \times \mathrm{SU}(4)$ to $U(1) \times \mathrm{SU}(3)$ occurs at some scale $m_{R} \sim 10^{11}-10^{13} \mathrm{GeV}$ with a unification scale where the three coupling constants meet of the order of $10^{16} \mathrm{GeV}$. All these breakings will have the Standard Model as an effective theory at low energies.

\section{Acknowledgments}

The work of AHC is supported in part by the National Science Foundation Phys- 1202671. WDvS would like to thank Gert Heckman for pointing to reference [20], and Florian Lyonnet for his help with PyR@TE. Also, WDvS thanks IHÉS for hospitality and support during a visit in June 2015 and NWO under VIDI-grant 016.133.326.

Open Access. This article is distributed under the terms of the Creative Commons Attribution License (CC-BY 4.0), which permits any use, distribution and reproduction in any medium, provided the original author(s) and source are credited.

\section{References}

[1] T. Appelquist and J. Carazzone, Infrared singularities and massive fields, Phys. Rev. D 11 (1975) 2856 [INSPIRE].

[2] U. Aydemir, D. Minic, C. Sun and T. Takeuchi, Pati-Salam unification from non-commutative geometry and the TeV-scale $W_{R}$ boson, arXiv:1509.01606 [INSPIRE].

[3] V.D. Barger, E. Ma and K. Whisnant, General analysis of a possible second weak neutral current in gauge models, Phys. Rev. D 26 (1982) 2378 [INSPIRE].

[4] A.H. Chamseddine and A. Connes, Universal formula for noncommutative geometry actions: unification of gravity and the Standard Model, Phys. Rev. Lett. 77 (1996) 4868 [INSPIRE].

[5] A.H. Chamseddine and A. Connes, The spectral action principle, Commun. Math. Phys. 186 (1997) 731 [hep-th/9606001] [INSPIRE].

[6] A.H. Chamseddine and A. Connes, Why the Standard Model, J. Geom. Phys. 58 (2008) 38 [arXiv:0706.3688] [INSPIRE].

[7] A.H. Chamseddine and A. Connes, Noncommutative geometry as a framework for unification of all fundamental interactions including gravity. Part I, Fortsch. Phys. 58 (2010) 553 [arXiv: 1004.0464] [INSPIRE].

[8] A.H. Chamseddine and A. Connes, Resilience of the spectral Standard Model, JHEP 09 (2012) 104 [arXiv:1208.1030] [INSPIRE]. 
[9] A.H. Chamseddine, A. Connes and M. Marcolli, Gravity and the Standard Model with neutrino mixing, Adv. Theor. Math. Phys. 11 (2007) 991 [hep-th/0610241] [INSPIRE].

[10] A.H. Chamseddine, A. Connes and V. Mukhanov, Geometry and the quantum: basics, JHEP 12 (2014) 098 [arXiv: 1411.0977] [INSPIRE].

[11] A.H. Chamseddine, A. Connes and V. Mukhanov, Quanta of geometry: noncommutative aspects, Phys. Rev. Lett. 114 (2015) 091302 [arXiv: 1409.2471] [INSPIRE].

[12] A.H. Chamseddine, A. Connes and W.D. van Suijlekom, Beyond the spectral Standard Model: emergence of Pati-Salam unification, JHEP 11 (2013) 132 [arXiv:1304.8050] [INSPIRE].

[13] D. Chang, R.N. Mohapatra and M.K. Parida, A new approach to left-right symmetry breaking in unified gauge theories, Phys. Rev. D 30 (1984) 1052 [INSPIRE].

[14] T.P. Cheng, E. Eichten and L.-F. Li, Higgs phenomena in asymptotically free gauge theories, Phys. Rev. D 9 (1974) 2259 [InSPIRE].

[15] V. Elias, Coupling constant renormalization in unified gauge theories containing the Pati-Salam model, Phys. Rev. D 14 (1976) 1896 [InSPIRE].

[16] V. Elias, Gauge coupling constant magnitudes in the Pati-Salam model, Phys. Rev. D 16 (1977) 1586 [INSPIRE].

[17] M.E. Machacek and M.T. Vaughn, Two loop renormalization group equations in a general quantum field theory. 1. Wave function renormalization, Nucl. Phys. B 222 (1983) 83 [INSPIRE].

[18] M.E. Machacek and M.T. Vaughn, Two loop renormalization group equations in a general quantum field theory. 2. Yukawa couplings, Nucl. Phys. B 236 (1984) 221 [InSPIRE].

[19] M.E. Machacek and M.T. Vaughn, Two loop renormalization group equations in a general quantum field theory. 3. Scalar quartic couplings, Nucl. Phys. B 249 (1985) 70 [InSPIRE].

[20] W. McKay and J. Patera, Tables of dimensions, indices, and branching rules for representations of simple Lie algebras, Lect. Notes Pure Appl. Math. 69, Marcel Dekker Inc., New York U.S.A. (1981).

[21] R. Mohapatra, Unification and supersymmetry. The frontiers of quark-lepton physics, third edition, Springer, New York U.S.A. (2003).

[22] J.C. Pati and A. Salam, Lepton number as the fourth color, Phys. Rev. D 10 (1974) 275 [Erratum ibid. D 11 (1975) 703] [INSPIRE]. 